\title{
Política farmacéutica saludable
}

\author{
Eduardo González Pier, D en Econ. ${ }^{(I)}$
}

\section{González-Pier E. \\ Política farmacéutica saludable. Salud Publica Mex 2008;50 supl 4:S488-S495.}

\section{Resumen}

Hoy en día, la industria farmacéutica se encuentra en una transición profunda. La globalización y el avance tecnológico representan las principales presiones de cambio para un mercado mundial de medicamentos donde a este tipo de industria le resulta cada vez más difícil recuperar de forma eficiente los costos crecientes de la innovación. México debe analizar las implicaciones en el ámbito político de estos factores de cambio y promover, en el mercado de medicamentos, una política que incremente al máximo las ganancias de salud de los recursos invertidos. La política de medicamentos ofrece un raro ejemplo de complementariedad entre una buena política de salud y una política económica eficiente, es decir, una "política farmacéutica saludable".

Palabras clave: industria farmacéutica; política farmacéutica; política nacional de medicamentos; innovación; México

\author{
González-Pier E. \\ Healthy pharmaceutical policy. \\ Salud Publica Mex 2008;50 suppl 4:S488-S495.
}

\begin{abstract}
Today, the pharmaceutical industry is experiencing a profound transition. Globalization and technological advancement represent the principal pressures for change in the market, where it is increasingly more difficult for this type of industry to efficiently recoup the growing cost of innovation. Mexico needs to analyze the policy implications of these change factors and promote, in the pharmaceutical market, policies that maximize health gains on invested resources. Pharmaceutical policy offers a rare example for a complementary approach between a sound health policy and an efficient economic policy; that is, a "healthy pharmaceutical policy."
\end{abstract}

Key words: drug industry; drug and narcotic control; national drug policy; innovation; Mexico
$\mathrm{P}$ ara analizar las principales características y tendencias mundiales de la industria es necesario, en primer lugar, abordar la estructura del mercado farmacéutico y de las políticas de precios que de ésta se desprenden. De entrada es importante distinguir entre dos tipos de empresas productoras de fármacos: aquellas enfocadas a la investigación y el desarrollo de nuevas moléculas que se traducen en medicamentos temporalmente protegidos por patentes, y las empresas que compiten en la producción de medicamentos genéricos una vez que expira la patente del ingrediente activo principal, generalmente después de 20 años de descubierto. ${ }^{1}$ La

Ensayo fundamentado en los mensajes de la Conferencia Magistral José Luis Bobadilla, impartida el 8 de marzo de 2007 en el marco del Congreso de Investigación en Salud Pública, del Instituto Nacional de Salud Pública, en Cuernavaca, Morelos, México.

(I) Ex titular de la Unidad de Análisis Económico, Secretaría de Salud. México.

Fecha de recibido: 9 de agosto de 2007 - Fecha de aceptado: 14 de marzo de 2008 Solicitud de sobretiros: Dr. Eduardo González Pier. Marina Nacional 329, col. Huasteca. I I3 I I, México, DF. Correo electrónico: egpier@gmail.com 
industria dedicada a la producción de nuevos medicamentos consiste en un reducido número de empresas transnacionales con sede principalmente en los Estados Unidos de América (EUA) y en Europa occidental, que compiten en un subconjunto de clases terapéuticas por un mercado mundial que en 2005 fue de cerca de $600 \mathrm{mil}$ millones de dólares. Esta industria es, precisamente, el foco de atención de este trabajo.

Una industria intensiva en investigación y desarrollo como la farmacéutica se distingue por un perfil de costos poco común. De acuerdo con estimaciones sobre la estructura de costos de la industria farmacéutica en los EUA, 31\% de los costos totales corresponden a actividades de investigación y desarrollo, $23.4 \%$ a mercadotecnia, y únicamente $28.2 \%$ a costos de producción. ${ }^{1}$ En términos generales $50 \%$ de los costos totales son costos hundidos, es decir, aquellos rubros de gasto que no son atribuibles a ningún mercado en particular. Esta característica de la industria farmacéutica es esencial para entender la política internacional de precios. ${ }^{2}$ Para recuperar costos hundidos y preservar los incentivos a la innovación, el precio que prevalece en la mayoría de los mercados de medicamentos es necesariamente mayor al costo marginal, es decir, aquel asociado de manera directa al costo de producción. Como parte complementaria de este equilibrio, el esquema de patentes, armonizado en la mayoría de los principales mercados del mundo mediante el Acuerdo sobre los Aspectos de los Derechos de Propiedad Intelectual relacionados con el Comercio (ADPIC, y más conocido por sus siglas en inglés: TRIPS) permite a los laboratorios una situación de monopolio artificial para cobrar un precio mayor al costo marginal durante el periodo de vigencia de la patente.

En este contexto de monopolio, el economista Frank Ramsey estableció desde 1927 que la forma más eficiente de recuperar costos -maximizando el bienestar socialbajo una situación de mercados segmentados, es fijar el margen del precio cobrado con respecto al costo marginal igual al inverso de la elasticidad precio de la demanda; al resultado se le ha llamado precios Ramsey., ${ }^{1,2}$ Ochenta años después, la evidencia internacional ha demostrado en términos generales la aplicación conceptual de precios Ramsey para medicamentos innovadores bajo patente. ${ }^{3-5}$ Con mayor o menor grado de detalle, esto significa que los países pobres pagan menos por los medicamentos bajo patente y los desarrollados pagan más -justo de forma aproximadamente proporcional a la capacidad de ingreso de cada país. ${ }^{*}$ Cabe señalar que otros determinantes del

\footnotetext{
* Otros determinantes del precio pueden ser la presencia de sustitutos terapéuticos, ahorros en el proceso terapéutico, aceptabilidad social, fragmentación de la compra y la existencia de mercados paralelos.
}

precio pueden ser la presencia de sustitutos terapéuticos, los ahorros en el proceso terapéutico, la aceptabilidad social, la fragmentación de la compra y la existencia de mercados paralelos.

Sin embargo, ése no ha sido el caso de México. Como consecuencia de la existencia de un comercio paralelo (mercados no segmentados) y de la compra fragmentada ( $80 \%$ del valor de las ventas de la industria farmacéutica se efectúa mediante gasto de bolsillo en el mercado privado), ${ }^{*}$ se observan precios relativamente altos; por ejemplo, en el mercado privado el precio promedio es tres veces mayor al equivalente en los EUA (ajustados por ingreso), cuando el PIB per cápita en ese país es seis veces mayor al de México. ${ }^{\ddagger}$ En la figura 1 se ilustra esta diferencia mediante la comparación de índices de precios para algunos países con respecto al nivel de precios y la canasta de consumo observada en los EUA. Dados los precios observados en México, si se invirtieran las proporciones considerando el valor y volumen de ventas de 2005, de manera que el sector público adquiriera $80 \%$ de las unidades vendidas -en vez de $20 \%$-, se generaría un ahorro estimado del orden de 40 mil millones de pesos. ${ }^{\S}$

\section{Retos de la industria ante la globalización}

Los aspectos de integración económica han resultado en una industria eminentemente global, a la vez que han hecho aun más compleja la tarea de conciliar los objetivos de eficiencia del mercado con el de mejorar las condiciones de salud. La globalización limita el potencial de las empresas para segmentar mercados y definir precios en cada uno de ellos de manera eficiente, es decir, de forma que se premie la innovación a la vez que el producto se comercializa en todos los mercados. Como resultado de estas tendencias, los comparativos de precios entre compradores, las prácticas de promoción e innovación y las experiencias tanto favorables como adversas en la implantación de nuevas regulaciones son compartidas entre pacientes y países de forma casi inmediata.

Un buen ejemplo de esta difícil interacción se observa en la reducción del costo de la información que representa el acceso a Internet. En muchos casos el

\footnotetext{
* Cifra estimada con base en información presentada por la Asociación Mexicana de Industrias de Investigación Farmacéutica, A.C. (AMIIF).

* Análisis elaborado por la Unidad de Análisis Económico de la Secretaría de Salud.

\& Cifra estimada con datos de la Asociación Mexicana de Industrias de Innovación Farmacéutica (AMIIF) sobre el valor total de ventas y unidades en los sectores privado y público en 2005.
} 


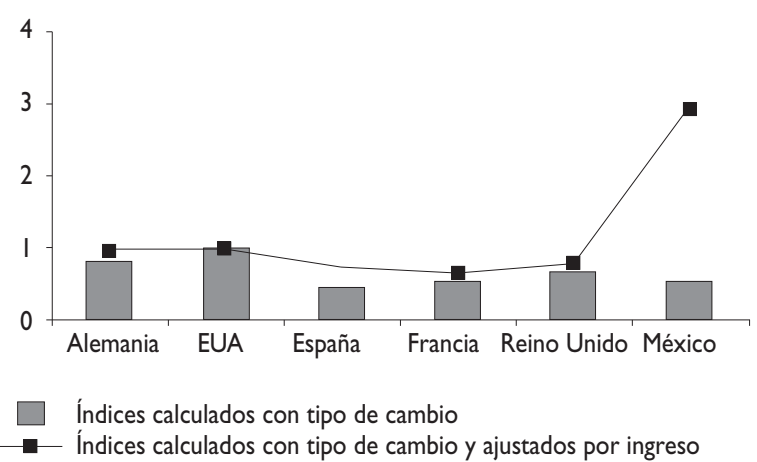

Fuente: Estimaciones de la Unidad de Análisis Económico, Secretaría de Salud, México

Figura I. ÍNDices de PRECIOS DE UNA CANASTA dE 22 MOLÉCULAs Bajo PATENTE (2005)

médico ha dejado de ser el informante principal de las alternativas terapéuticas y farmacológicas del paciente. El crecimiento de la publicidad de la industria dirigida de forma directa a los pacientes y la disponibilidad de información clínica a la que ahora puede acceder directamente la población, han generado pacientes que cuestionan cada vez más a sus médicos y exigen la cobertura de nuevas terapias. Ante la necesidad de acomodar estas presiones de servicio y de evitar el aumento en los costos de la atención médica, las instituciones aseguradoras de salud han tenido que presionar a la baja los costos de atención y los precios de sus principales insumos. Un ejemplo de este fenómeno es la compra transfronteriza de medicamentos en Canadá que realizan los gobiernos estatales de los EUA a través de detallistas por Internet, así como el caso de la frontera con México, donde se estima que aproximadamente 5\% de los más de 100 millones de cruces anuales entre Tijuana y San Diego son por razones de atención médica, principalmente para la compra de medicamentos.

En el caso de medicamentos, las iniciativas de contención del gasto se traducen en presiones para regular directamente los precios o controlarlos de manera indirecta a través del uso de precios de referencia para el reembolso máximo del costo de los medicamentos. Esto conlleva a un mayor escrutinio de la efectividad relativa entre medicamentos y otras alternativas terapéuticas, y a promover la introducción oportuna y el uso más generalizado de medicamentos genéricos.

De manera reciente se ha observado la práctica de regular los precios máximos de medicamentos bajo patente con base en la referencia cruzada de los precios de canastas de los mismos medicamentos en otros mercados. En la medida en que las autoridades de distintos países refieran los precios de forma cruzada entre sí, la industria perderá la capacidad de segmentar los mercados y diferenciar sus precios en función de las condiciones locales de la demanda; en consecuencia, el sistema se moverá hacia precios más uniformes. Así por ejemplo, los precios de los medicamentos observados en el Reino Unido, cuyo mercado representa sólo 4\% del mercado mundial, tienen un efecto sobre $25 \%$ del mercado -principalmente de otros países de Europa que utilizan el precio británico como referente. ${ }^{6}$ Esta tendencia, paradójicamente, beneficia a los mercados con precios más altos en detrimento de los mercados de países en vías de desarrollo como México, al tiempo que reduce el bienestar social, pues el uso de precios uniformes impide recuperar los costos de las actividades de investigación y desarrollo de forma eficiente.

En consecuencia, al mismo tiempo que los laboratorios buscan formas de recuperar costos de inversión en investigación y desarrollo, se observa una presión creciente de grupos de pacientes y organizaciones no gubernamentales por flexibilizar el orden mundial de respeto a la propiedad industrial para permitir el acceso de copias de medicamentos bajo patente asociados a enfermedades graves y riesgos de seguridad nacional. Esto explica varias iniciativas recientes para promover el licenciamiento obligatorio de productos y condicionar el apego a la protección de los derechos de propiedad industrial en función de otras contingencias. En octubre 2001, por ejemplo, como parte de la estrategia para contener un posible ataque de ántrax, el gobierno federal de los EUA persuadió a Bayer, titular de la patente del antibiótico ciprofloxacino, a reducir significativamente su precio para evitar se adquiriera copias del medicamento en otros laboratorios. El precio resultante de la negociación fue 47\% menor: 95 centavos de dólar por tableta para un pedido inicial de 100 millones de tabletas, en comparación con el precio anteriormente pagado de 1.77 dólares. ${ }^{7}$

En el mismo sentido, después de las presiones ejercidas a través del comunicado de prensa del senador norteamericano Charles E. Schumer en octubre de 2005 y de las declaraciones de Kofi Annan, Secretario General de la $\mathrm{ONU}, 8,9$ ante la amenaza de una pandemia de gripe aviar, al final de 2005 Roche tuvo que negociar con compañías externas el permiso para que pudieran producir oseltamivir, el componente activo del Tamiflu, su medicamento para combatir la gripe aviar. No menos significativas son las presiones por parte de países en desarrollo, como lo ilustran los casos de Brasil y Sudáfrica en torno al tratamiento de antirretrovirales para combatir el virus de inmunodeficiencia humana para incorporar exenciones al ADPIC y motivar el replan- 
teamiento de la política ejercida por los gobiernos en la materia.

Finalmente, como uno de los principales retos y críticas que enfrenta la industria, destaca el hecho de que a pesar de observarse un flujo constante de entrada de nuevos productos, muchos son menos innovadores y en realidad constituyen meras innovaciones marginales o copias modificadas de otros productos con patente. Se estima que sólo una tercera parte de las nuevas moléculas introducidas al mercado constituyen una innovación significativa; el resto se consideran innovaciones marginales desde el punto de vista terapéutico (a estos medicamentos se les conoce también como me-too drugs).

El área en donde parece concentrarse de manera creciente el flujo de innovación efectiva es la biotecnología, no sólo como resultado de los avances significativos en genómica, sino también en el desarrollo de la fisiología celular y otras áreas de ciencia especializada, y de tecnologías que permiten procesar y sintetizar sustancias a partir de compuestos orgánicos. El desarrollo de medicamentos innovadores en esta área genera nuevos retos en materia de protección de la propiedad intelectual, como es la necesidad de regular mejor el otorgamiento de patentes a procesos y métodos de aplicación, y no necesariamente a nuevas moléculas.

\section{La industria en transición}

Ante este contexto de retos emergentes, se pueden observar tres tendencias recientes que ejercen presión sobre la organización actual de la industria:

Primero, la presencia y competitividad de la industria de genéricos con una creciente presencia trasnacional. Por ejemplo, en Canadá, uno de los países con mayor penetración de genéricos, la participación de estos medicamentos en el surtimiento ambulatorio de recetas ha aumentado de $26.2 \%$ en 1990 a $44.5 \%$ en 2006 . A su vez, la participación de genéricos en las compras de hospitales y farmacias ha pasado de 9.3 a $18.1 \%$ en el mismo periodo, ${ }^{10}$ mientras que en los EUA, entre 1984 y 1996, la participación de mercado de medicamentos genéricos aumentó de poco menos de 20\% a algo más de 40\%. ${ }^{11-13}$ Esta tendencia se verá reforzada ante el hecho de que 12 de los 35 medicamentos de mayor venta en el mundo perderán la protección de patente antes de 2010.

A manera de ejemplo de la dura competencia entre estas empresas cabe mencionar que, en agosto de 2006, la Corte Federal de Apelaciones de los EUA falló a favor de Ranbaxy (empresa productora de genéricos de la India) y adelantó la fecha de vencimiento de Lipitor (atorvastatina) de junio 2011 a marzo 2010. ${ }^{14,15}$ Se trata aquí del medicamento de mayor venta en el mundo -12.9 billones de dólares en 2006- y representa 40\% de las utilidades de Pfizer, la empresa más grande de esta industria. Esto ha generado todavía más presiones para Pfizer, que en diciembre de 2006 tuvo que suspender los ensayos clínicos de torcetrapib (el sucesor de Lipitor) debido al "desequilibrio observado en mortalidad y eventos cardiovasculares" en relación con su consumo. 16,17

La ventaja competitiva de la industria de genéricos radica en su capacidad de ofrecer productos de probada seguridad y eficacia a un costo más bajo, al evitarse los costos hundidos asociados a la inversión en actividades de investigación y desarrollo. Ante la necesidad de contener el gasto en atención médica, es probable que la participación de estos productos aumente en la medida en que los esquemas de aseguramiento médico de los diversos sistemas de salud sigan la tendencia de favorecer su financiamiento, siempre y cuando éstos representen una opción más costo-efectiva con respecto a los medicamentos innovadores.

Segundo, también se observa un incremento significativo en el costo que implica la creación y la introducción al mercado de nuevos medicamentos. En 2005 el gasto total en actividades de investigación y desarrollo se estimó en 55 mil millones de dólares, el doble de lo registrado en 1993. Sin embargo, en 2005 solamente se introdujeron 25 nuevas moléculas, en comparación con la introducción en 1993 de 40 nuevas moléculas (figura 2). Esta aparente reducción en el retorno de la inversión en investigación y desarrollo ha llevado a la industria a citar que cada medicamento cuesta entre 500 y 2000 millones de dólares, aunque algunas estimaciones puntuales citan un costo de 800 millones de dólares. ${ }^{18,19}$

Tercero, de acuerdo con evidencia para los EUA, la opinión pública sobre la industria se ha deteriorado de forma considerable. Como se aprecia en la figura 3 , inclusive cuando la percepción sobre la industria es positiva en términos netos y con respecto a otras industrias, entre 1998 y 2005 se observó un deterioro significativo. Para explicar lo anterior baste recordar tres casos que causaron gran revuelo en los medios de comunicación: 1) el retiro del antiinflamatorio Vioxx; ${ }^{20}$ 2) la demanda que presentara el entonces fiscal y ahora gobernador del estado de Nueva York Eliot Spitzer contra GlaxoSmithKline por el supuesto encubrimiento de datos que asociaban el uso de un antidepresivo con la tendencia de suicidios en jóvenes, ${ }^{21}$ y 3 ) el retiro unilateral por razones de precios y debates de carácter moral, a principios de 2007, por parte de Merck de sus esfuerzos de cabildeo en los congresos locales de los EUA para promover la compra de su nueva vacuna contra el virus del papiloma humano. ${ }^{22}$ 


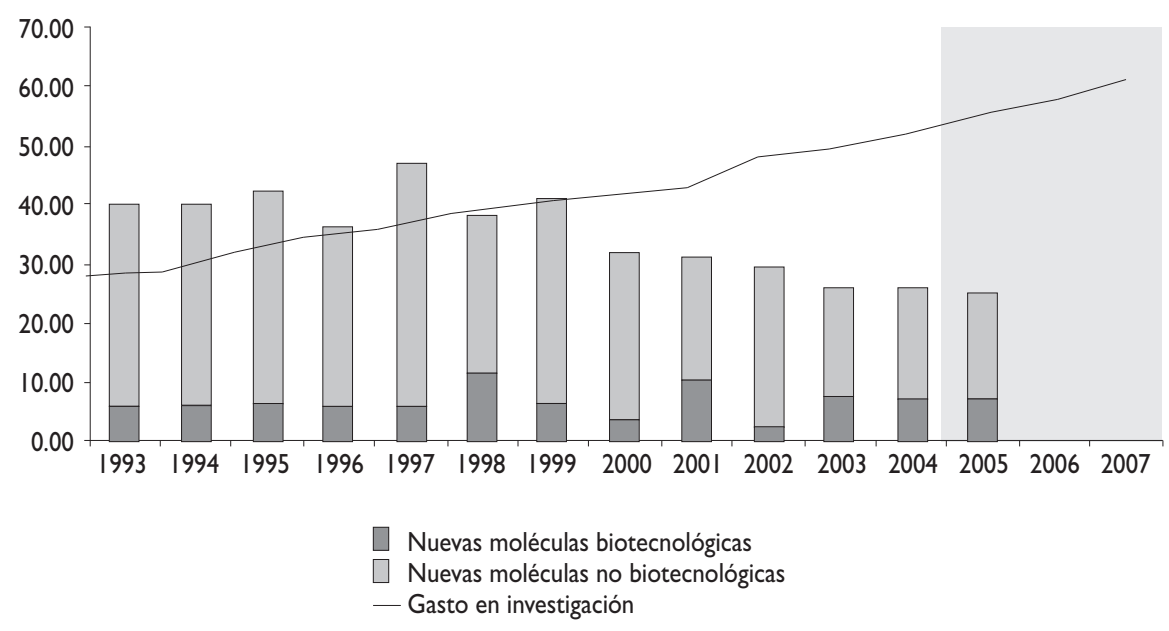

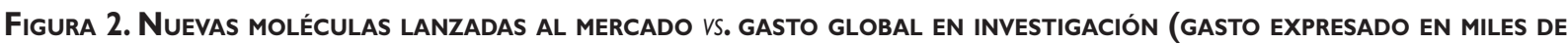
MILLONES DE DÓLARES)

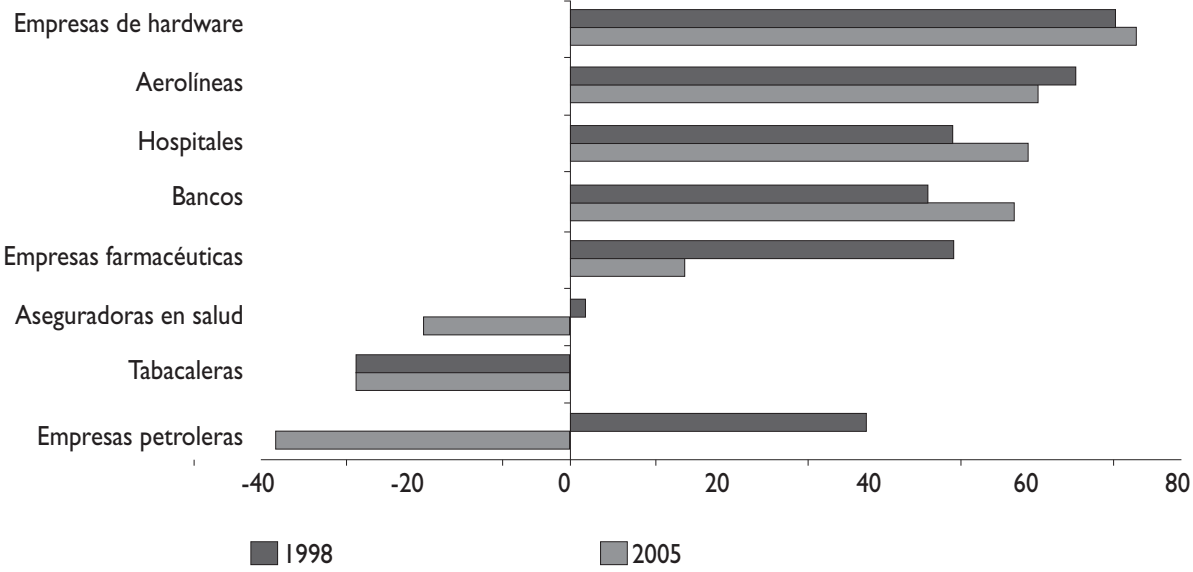

Figura 3. Percepción del servicio proporcionado al consumidor por tipo de empresa (Balance de "Buen Servicio" MENOS “MAL SERVICIO")

\section{Implicaciones de política para México}

Antes de pasar a las conclusiones sobre las implicaciones de política para el caso de México -y posiblemente para cualquier otro país de ingreso medio-, es importante entender la evolución de dos etapas muy distintas en la política y el desarrollo de la industria farmacéutica en nuestro país:
La conformación del sistema moderno de salud, 1943-1990

Durante este periodo que inicia con la puesta en marcha del actual sistema nacional de salud, primero con la creación del Instituto Mexicano del Seguro Social (IMSS) y de la Secretaría de Salud (entonces Secretaría de Salubridad y Asistencia) en 1943, y hasta poco antes de la entrada de México al Tratado de Libre Comercio de 
América del Norte (TLCAN), el mercado se caracterizó por la comercialización de productos conocidos como "copias" -el uso de patentes con vigencia de 20 años se introdujo en México hasta 1991-y por el fomento de una industria nacional proveedora para el sector gobierno. En contraste, el mercado privado se desarrolló enfocando sus ventas a productos de marca (y eventualmente de patente), que eran comercializados en el sector privado y pagados, sobre todo, mediante el gasto de bolsillo de los hogares.

La industria se concentró en la provisión de vacunas, antibióticos y otros productos necesarios para responder a un perfil epidemiológico principalmente vinculado con enfermedades infecciosas. En esta etapa, la industria nacional era la primera fuente de abasto del sector público, en particular para el IMSS, y los laboratorios nacionales invertían sobre todo en la reproducción de moléculas existentes, con pocos esfuerzos en investigación y desarrollo para promover actividades locales de innovación. Hubo pocos casos exitosos de innovación generada en México, como fue el desarrollo de la progesterona sintetizada a partir del barbasco, que llevó a la fundación de la empresa Syntex en 1944. De igual manera, durante este periodo cobró fuerza la presencia de fármacos orientados a combatir enfermedades crónico-degenerativas. Más tarde, hacia la década de los noventa, se introdujeron en México y en el mundo nuevas clases de medicamentos para padecimientos emergentes como las estatinas para el control de dislipidemias y los inhibidores selectivos de recaptura de serotonina para tratar la depresión clínica.

\section{La apertura de México en el ámbito internacional, 1990-2004}

Como parte de las políticas necesarias para incorporar al país al TLCAN, hubo cambios radicales en la regulación del sector, entre ellos las medidas necesarias para garantizar la protección de la propiedad industrial. En 1991 se publicó la Ley de Fomento y Protección de la Propiedad Industrial (hoy Ley de la Propiedad Industrial) mediante la cual se otorgan patentes con vigencia de 20 años. En 1995 México se adhirió a la Organización Mundial del Comercio y quedó sujeto al ADPIC.

A manera de una renovación regulatoria importante, en 1998 se publicó el Reglamento de Insumos para la Salud y se introdujo el programa de genéricos intercambiables. Al mismo tiempo, conforme el sector público aumentaba la compra de medicamentos de patente, se desvanecían los binomios: mercado privado/ medicamentos de patente y mercado público/ medicamentos genéricos. En 2001 se creó la Comisión Federal para la Protección contra Riesgos Sanitarios (COFEPRIS) como instancia desconcentrada de la Secretaría de Salud, con lo que se fortaleció el papel del Estado en la regulación sanitaria. En 2004 se actualizó el esquema de regulación directa de precios en el sector privado ejercido por la Secretaría de Economía en el marco del Programa de Modernización de la Industria Farmacéutica en Materia de Precios (PROMIF). Bajo la nueva regulación y ante la falta de evidencia que justificara, bajo principios económicos, su control, se liberaron los precios máximos de venta al público de medicamentos genéricos. En cambio, se mantuvo el control de los precios máximos al menudeo de medicamentos con patente, mismo que se establece en función de los precios observados en los seis principales mercados de cada producto sujeto a la regulación. Por último, durante este periodo se introdujeron al mercado nuevos medicamentos fundamentales para el tratamiento de enfermedades crónicas, y aumentó el número de estatinas, inhibidores ACE y otros medicamentos del grupo cardiovascular y de medicamentos oncológicos en correspondencia a un nuevo perfil de mortalidad asociado a enfermedades no contagiosas.

Sin embargo, desde 2005 se observa un aumento de precios en el mercado privado y una mayor presión en el gasto público en aseguramiento, en particular para el tratamiento de enfermedades crónico-degenerativas (sobre todo diabetes e hipertensión). Para lo que se podría considerar como el inicio de una nueva etapa, se prevén también cambios en la configuración del aseguramiento médico en México, en la medida en que iniciativas de aseguramiento en salud como el Seguro Popular de Salud se consoliden y tengan, a su vez, repercusiones importantes en la estrategias de abasto, dispensación y composición del mercado farmacéutico en términos de las participaciones del sector público y privado, tanto en valor como en volumen.

Dados los antecedentes y a la luz del contexto que enfrenta la industria farmacéutica en el mundo, México se encuentra ante la oportunidad de sumarse a nuevas iniciativas globales para mejorar el desempeño de la industria y sus beneficios sobre la salud de la población. Por un lado es necesario que se sume al esfuerzo internacional de premiar a una industria de innovación auténtica, es decir evitar las copias terapéuticas (me-too drugs). Las tendencias en la regulación de precios de productos innovadores apuntan a reconocer la necesidad de pagar mayores precios por el valor de los beneficios de la innovación, pero sólo cuando la innovación demuestre generar en el margen mayores ganancias en salud por cada peso invertido. ${ }^{5}$ En México se podría promover ese tipo de innovación mediante una política de precios en el sector público en donde se pague por los medicamentos innovadores en función de su grado de innovación, el cual ha de medirse a través de los beneficios terapéuticos observados para los pacientes. 


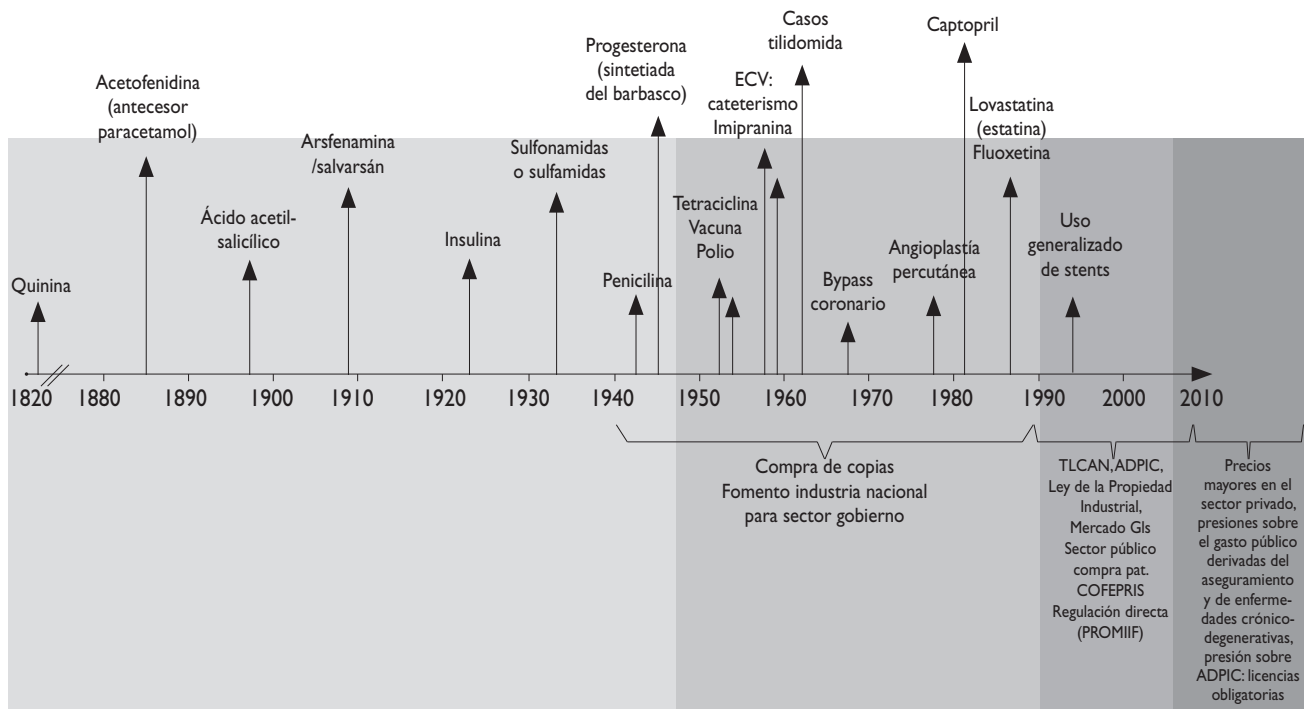

ECV: enfermedad cerebro-vascular

Figura 4. Algunos hitos en la evolución del mercado farmacéutico y en la política farmacéutica en MéXico

Lamentablemente, la regulación en México se ha restringido al control arbitrario de precios sin vinculación alguna con una mejor comprensión del funcionamiento del mercado, y sin tomar en cuenta la heterogeneidad de productos disponibles o la necesidad de definir una regulación que considere el funcionamiento del mismo. Si bien los medicamentos genéricos son más baratos y es deseable fomentar su consumo, es necesario promover e institucionalizar los instrumentos de evaluación necesarios para discernir cuando la terapia innovadora genera una reducción en costos en otros rubros de la atención médica (estancias hospitalarias, atención de efectos secundarios, entre otras) y encontrar un mejor balance de gasto entre medicamentos innovadores y genéricos. A este respecto, estimaciones realizadas por Frank Lichtenberg indican que el lanzamiento de nuevas moléculas explica 40\% del aumento en la longevidad observada en el periodo 1986-2000.23

Las alternativas de política para los próximos 20 o 30 años son pocas. México puede mantenerse con un alcance limitado de la política farmacéutica reducido a lograr precios lo suficientemente bajos para mejorar el acceso, pero lo suficientemente altos para promover la entrada oportuna de nuevas terapias. Esto implicaría seguir dependiendo de la innovación en otros países -en ocasiones sustentada en poblaciones con perfiles epidemiológicos distintos y, más grave todavía, a partir de un perfil genético distinto al de la población mexica- na, precisamente cuando el futuro apunta a la medicina personalizada basada en la farmacogenómica.

Sin embargo promover la innovación local sería el camino deseable para que el desarrollo de nuevos productos atienda el perfil de enfermedades y el perfil genético específico de los mexicanos. Empero, el respeto a la protección de la propiedad intelectual debe ser visto como una condición necesaria pero no suficiente para promover innovación que corresponda a las necesidades de la población mexicana. Se requiere necesariamente analizar y aprender de las experiencias exitosas en el plano internacional, mismas que apuntan hacia nuevos modelos legislativos y financieros para alinear los incentivos a la producción de ciencia básica y el usufructo directo de beneficios a través de patentes otorgadas a instancias de gobierno y universidades. Asimismo se insiste en promover con recursos públicos una mayor inversión en capital humano y, en lo posible, la repatriación de científicos mediante mejores oportunidades profesionales e incrementos significativos en inversión, en equipo e infraestructura. Muchas de estas políticas, que empiezan a dar resultados en otros países (por ejemplo en Irlanda, Australia y Singapur), desafortunadamente se encuentran muy lejanas de la situación que impera actualmente en México. ${ }^{24} \mathrm{El}$ reto es grande, pero el costo de no vincular los objetivos y la organización del sistema de salud con una política farmacéutica congruente con las necesidades de la población puede ser todavía mayor. 
México debe entender las implicaciones de política de estas fuerzas de cambio y promover una política en el mercado de medicamentos que maximice las ganancias de salud por cada peso invertido, procurando la complementariedad entre una buena política de salud y una política económica eficiente, es decir una "política farmacéutica saludable".

\section{Agradecimientos}

Se agradece la participación y las valiosas contribuciones de Mariana Barraza Lloréns en la elaboración de este ensayo.

\section{Referencias}

I. Danzon PM. Pharmaceutical price regulation: National policies versus global interests. Washington DC:AEl Press, 1997.

2. González-Pier E, González-Hernández A. Regulación saludable del sector farmacéutico. En: Comisión Federal de Competencia. Competencia Económica en México. México: Porrúa, 2004.

3. Puig-Junoy J, ed. The public financing of pharmaceuticals. An economic approach. Cheltenham: Edward Elgar Publishing, 2005.

4. Danzon PM, Furukawa MF. Prices and availability of pharmaceuticals: Evidence from nine countries. Health Aff 2003; 22(6):W52I-W536.

5. Danzon PM, Furukawa MF. Prices and availability of biopharmaceuticals: An international comparison. Health Aff 2006; 25(5): I 353-I 362.

6. Office of Fair Trading. The Pharmaceutical Price Regulation Scheme.An OFT market study. London: OFT, 2007.

7. United States Department of Health and Human Services. HHS, Bayer agree to Cipro purchase. HHS Press Release. [Accesed on: October 24,200I]. Available from: www.hhs.gov/news.

8. United States Senate. Letter from US Senator Charles Schumer to Dr. Franz B. Humer, CEO of F. Hoffman-La Roche Ltd. [Accesed on: October 17, 2005]. Available from: www.senate.gov.

9. Kanter J. Roche offers to negotiate on flu drug. The New York Times. [Accesed on: October 19, 2005]. Available from: www.nytimes.com.
10. Canadian Generic Pharmaceutical Association. Available from: http:// www.canadiangenerics.ca/en/resource/market_trends.shtml II. Congressional Budget Office. How increased competition from generic drugs has affected prices and returns in the pharmaceutical industry. CBO Publications 1998; Available from: www.cbo.gov. 12. Office of Fair Trading. Annexe D - Global overview of the pharmaceutical industry. En: Office of Fair Trading. The Pharmaceutical Price Regulation Scheme 2007; Available from: www.oft.gov.uk. 13. Mossialos E, Mrazek M,Walley T eds. Regulating pharmaceuticals in Europe: striving for efficiency, equality and quality. Berkshire: Open University Press, 2004.

I4. Billion dollar pills. The Economist 2007 enero 25;Available from: www. Economist.com.

15. Bloomberg News. Court invalidates a patent that Pfizer holds for Lipitor. The New York Times 2006 agosto 3; Available from: www.nytimes. com

16. Food and Drug Administration. Pfizer Stops All Torcetrapib Clinical Trials in Interest of Patient Safety. FDA Statement 2006 diciembre 3; Available from: www.fda.gov.

17. Berenson A. End of Drug Trial Is a Big Loss for Pfizer. The New York Times 2006 diciembre 4; Available from: www.nytimes.com.

18. Testing times. The Economist 2005 junio 16; Available from: www. Economist.com.

19. DiMasi JA, Hansen RW, Grabowski HG.. The price of innovation: new estimates of drug development costs. J Health Econ 2003; 22:15I- 185.

20. Food and Drug Administration. FDA Issues Public Health Advisory on Vioxx as its Manufacturer Voluntarily Withdraws the Product. FDA News 2004 septiembre 30; Available from: www.fda.gov.

21. Harris G. Spitzer Sues a Drug Maker, Saying It Hid Negative Data, The New York Times 2004 junio 3; Available from: www.nytimes.com. 22. Pollack A, Saul S. Merck to Halt Lobbying for Vaccine for Girls. The New York Times 2007 febrero 21; Available from: nytimes.com. 23. Lichtenber FR. The impact of New Drug Launches OA Longevity: evidence from longitudinal, Disease-Level data from 52 countries 1982-200I. International Journal of Health Care Finance and Economics 2005;5:47-73.

24. Watson E. Pharmaceutical research and development in New ZealandOn the brink of the abyss. New Zealand: Nazadel Ltd Biotechnology and Pharmaceutical Consultants, 2006. 